\title{
IMMUNOASSAY FLOW SYSTEMS ON-CHIP
}

\author{
D. Jed Harrison, Nghia Chiem \\ Department of Chemistry, University of Alberta, Edmonton, Alberta, Canada, T6G 2G2
}

\begin{abstract}
Integrated microfluidic systems for biochemical analyses and immunoassays were prepared in glass substrates using silicon micromachining techniques. All of the fluid handling steps are performed by applied electric fields, eliminating the need for pumps and microvalves. Electroosmotic pumping was used to drive immunological reagents and samples into a separation channel and the reaction products of the immuno-reaction were evaluated. The extent of reaction can be used to quantitate the amount of sample present when uncomplexed reagents are separated from the product complex. Concentration detection limits of about $30 \mathrm{pM}$ were obtained for fluorescein using fluorescence detection. Detection of the drug theophylline at 5 $\mathrm{ng} / \mathrm{ml}$ was shown in an immunoassay. Immunoassays for the large proteins bovine serum albumin and human immunoglobulin were also performed on-chip.
\end{abstract}

\section{INTRODUCTION}

Microfluidic devices etched in glass substrates provide a fluidic network in which chemical reactions, sample injection and separation of reaction products can be achieved. The application of high voltages to conductive fluids within these channels leads to electroosmotic and/or electrophoretic pumping, providing both mass transport and separation of components within the samples. In this paper we present on-chip, clinically relevant, immunological assays for proteins such as albumin, and drugs such as theophylline. Electroosmotic pumping is used to deliver reagents and samples, and separation of the reaction products is performed using electrophoresis [1]. This method pumps fluid at velocities up to $1 \mathrm{~cm} / \mathrm{s}$ in approximately $20 \mu \mathrm{m}$ diameter capillaries, while also controlling the direction of fluid flow at capillary intersections, without a need for valves or other moving parts. Electrophoretic effects lead to separation: i.e. the differing mobilities of ions result in different migration rates within an electric field, giving rise to separations.

Application of electrophoresis to immunoassays is a newly developing field [2-5]. Antibodies (Ab) are highly selective biological molecules originating from the immune system, capable of binding with high affinity to a particular target molecule known as an antigen (Ag). Immunologically based assays provide a common method to selectively determine many chemicals at low concentration. In common immunosorbent assays, in which the reagents are adsorbed onto surfaces, there are many complex fluid handling steps involved. We expect that the unique fluid delivery capabilities of the micro-electrophoresis chip format will provide a novel, important new method of automating immunoassays in a manner which does not involve the conventional robotics used in clinical labs today. The chip format will also provide much more rapid presentation of results for samples in clinical and environmental applications. The key step towards developing this technology illustrated in this paper is the separation of products and reactants resulting from homogeneous immunoassays, and the demonstration of quantitative analysis of the targeted sample.

\section{EXPERIMENTAL}

Devices were fabricated in Pyrex glass or a photomask crown glass [6]. The potential control and measurement instrumentation has also been presented previously [6]. Buffers were adjusted to $\mathrm{pH} 8$ or 9 . Antibodies, other proteins, and the fluorescent labels were purchased from Sigma. The theophylline reagents used were part of the Abbott TDx reagent set.

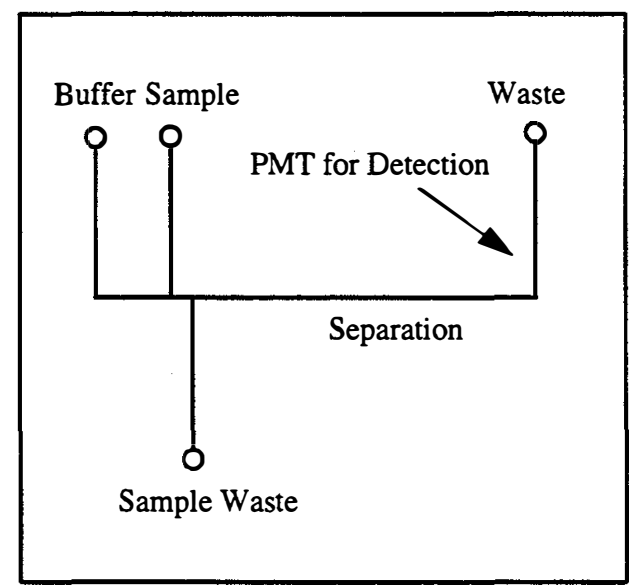

Figure 1 Layout of the flow channels used for immunoassay chip.

\section{RESULTS AND DISCUSSION}

Integrated devices for chemical separation employed in this study are based on capillary electrophoresis (CE), a method in which high applied voltages can be used to separate the components of a sample with high efficiency. Figure 1 illustrates the layout of the fluid channels in the chip used for these studies. Sample is introduced through a side channel and driven towards sample waste with 500 to $1500 \mathrm{~V}$, creating a $60-100 \mathrm{pL}$ plug of solution at the intersection, which can be injected into the separation channel. Fluorescence detection is used with a $488 \mathrm{~nm}$ laser excitation source and a photomultiplier tube for detection of 
the emission from fluorescently labelled molecules. This detection system is highly selective, as only fluorescent compounds will be observed [7].

Sensitivity is a key issue in immunoassays, as most of the compounds of interest are present at very low concentrations. We have improved detection substantially since earlier reports, by optimizing the wafer bonding process to reduce formation of light scattering centers on the surface of the glass devices. Careful alignment of the laser beam to eliminate scatter from the curved walls of the channels was also beneficial. In this way we have reduced our previous detection limits from about $2 \mathrm{nM}$ down to 30 pM. For the optical cell geomerry used, this detection limit corresponds to about 200 molecules in the detection volume. Figure 2 shows the signal obtained for 100 pM fluorescein, which gives a signal to noise ratio of 14 . Detection limits in the $\mathrm{pM}$ range are salisfactory for a number of immunoassays.

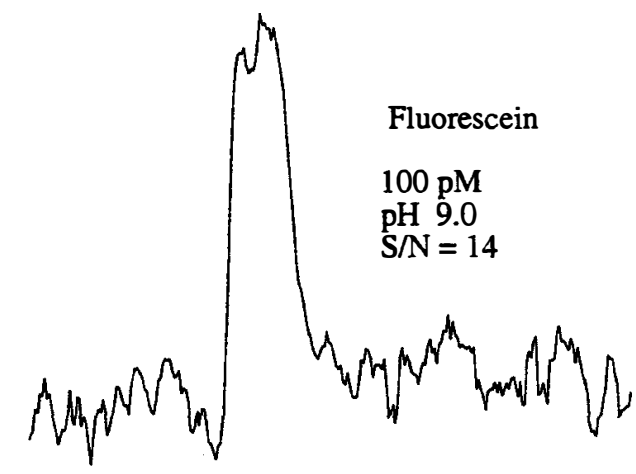

Figure 2 Fluorescence signal from 100 pM fluorescein in the chip.

Homogeneous immunoassays are solution phase reactions of an antibody and its a target molecule, the antigen. By labelling either the antigen $(\mathrm{Ag})$ or the antibody $(\mathrm{Ab})$ with a fluorescent tag, it is possible to determine when a complex between antibody and antigen has been formed. (We will designate labelled compounds with $a^{*}$.) Figure 3 shows an on-chip separation of a labelled antibody $\left(\mathrm{Ab}^{*}\right)$ before and after reaction with its antigen. In this case the antigen is human immunoglobulin $G$ (IgG, itself an antibody), while the antibody is fluorescently labelled goat antihuman IgG. The complexes formed between the two compounds have a different charge to size ratio than either of the free compounds, so they migrate at different rates in the electric field. The unlabelled 160,000 Dalton target, human IgG, can be determined from its complex formation with the anti-human antibody, while the remaining free antibody is separated from the reacted material. Figure 3 shows there are two product peaks, representing formation of both the $A b^{*}-A g$ and $A b^{*}-A_{2}$ complexes, as the antibody is bivalent.

Figure 4 illustrates an immunoassay involving a lower molecular weight protein than in the above study. This is an assay for the antibody to the 60,000 Dalton protein bovine serum albumin (BSA). To determine the amount of antibody present, it is mixed with $50 \mu \mathrm{g} / \mathrm{ml}$ of flourescently labelled BSA* and

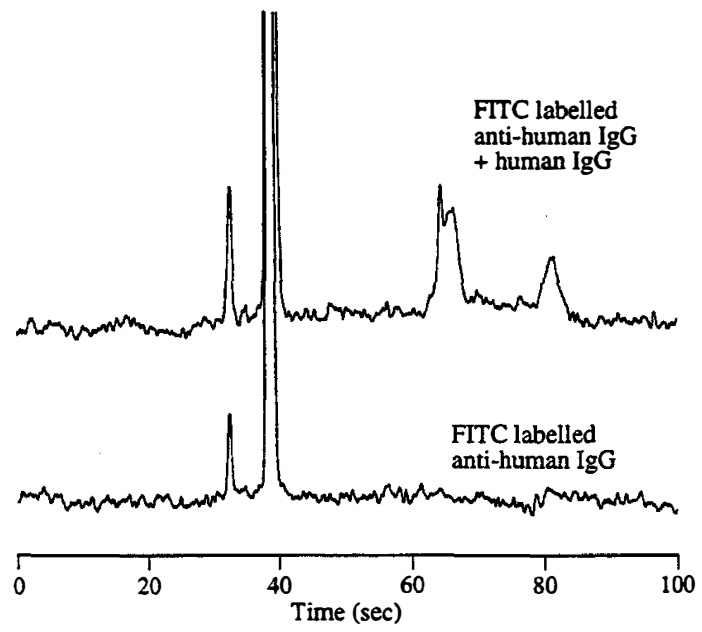

Figure 3 Bottom trace shows separation of fluorescein labelled anti-human IgG. Top trace shows the separation after addition of human $\operatorname{Ig} G$.

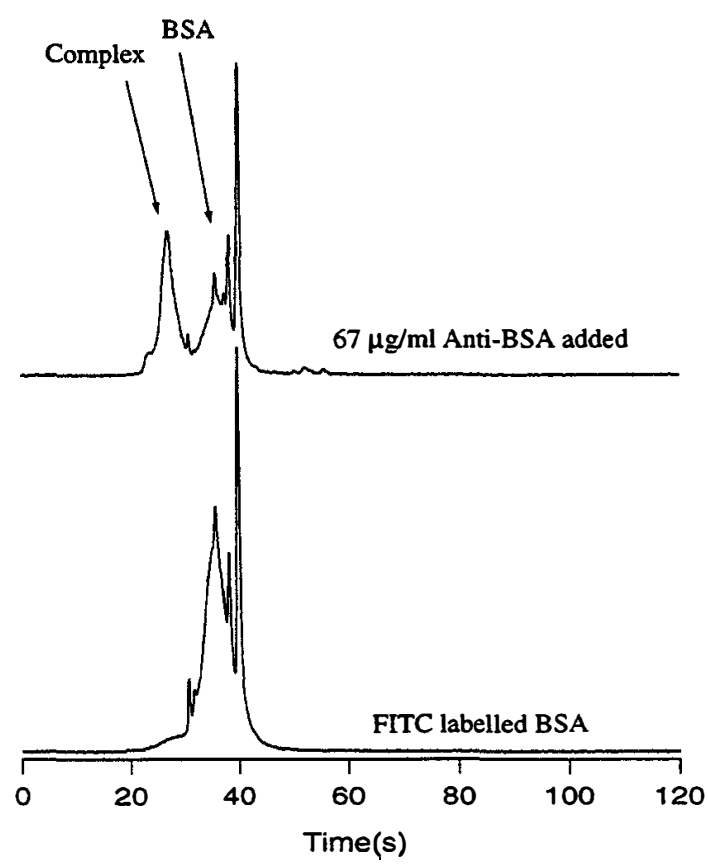

Figure 4 Bottom trace shows separation of Fluorescein labelled $B S A$, with a number of impurities present in the commercial product. Top trace shows the separation after addition of mouse ascites fluid containing antiBSA.

separated. The complex formed migrates at a different rate, and is separated from the free BSA*. The result is a decrease in signal for $\mathrm{BSA}^{*}$ and an increase in the Ab-BSA* complex peak. The increase in peak height or area is proportional to the amount of anti-BSA present.

Figure 5 shows a calibration curve for the assay of BSA antibody. In this study the anti-BSA source was a solution of mouse ascites fluid. This fluid is the extraction fluid as obtained directly from mice during monoclonal antibody harvesting. 


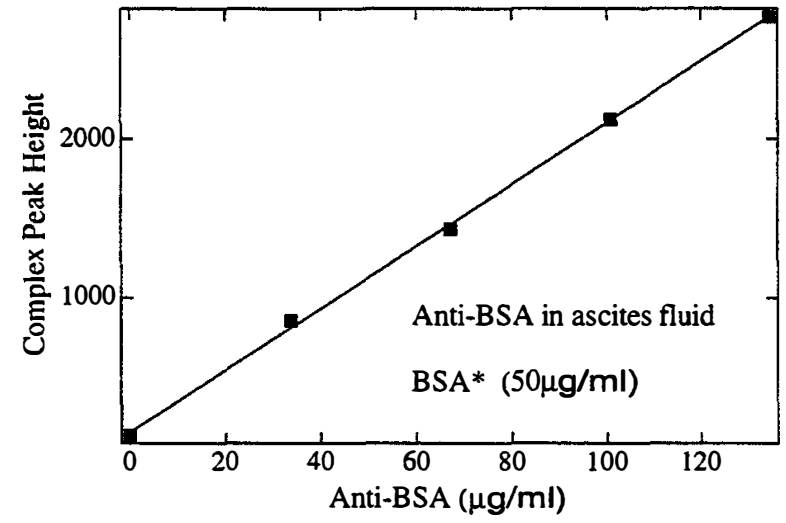

Figure 5 Calibration curve for anti-BSA in ascites fluid, measured from the complex peak height.

It is rich in a variety of proteins $(27 \mathrm{mg} / \mathrm{ml})$, of which only $10 \%$ is the desired antibody. The fact that good separations and quantitative assays can be obtained from this complex fluid illustrates the robustness of the microchip-CE system for these assays. Deliberate addition of other antibodies, which are not specific for BSA, showed no complex formation, indicating the selectivity of the antibody reaction. When combined with the separation power of $\mathrm{CE}$, immunoassay on-chip makes for a very powerful, selective immunosensor system, capable of identifying specific chemicals or biochemicals in a complex sample mixture.

It is also of great interest to analyze for small molecules by immunoassay methods. A typical example is an analysis for a therapeutic drug for asthma, theophylline (Th). In order to get good separations by $\mathrm{CE}$ when using small target molecules, it is necessary to label the analyte molecule instead of the antibody. Labelled $\mathrm{Th}\left(\mathrm{Th}^{*}\right)$ is thus mixed with a sample containing unlabelled Th, and the two are allowed to compete for a limited amount of antibody to Th, as illustrated in the scheme below.

$$
\begin{gathered}
\mathrm{Ab}+\mathrm{Th}^{*} \Leftrightarrow \mathrm{Ab}-\mathrm{Th}^{*} \\
\mathrm{Ab}+\mathrm{Th}+\mathrm{Th}^{*} \Leftrightarrow \mathrm{Ab}-\mathrm{Th}+\mathrm{Ab}-\mathrm{Th}^{*}
\end{gathered}
$$

A competitive assay leads to an increase in signal for the free, labelled $\mathrm{Th}^{*}$ as $\mathrm{Th}$ from a sample increases in concentration. There is a corresponding decrease in signal from the Ab-Th* complex. Figure 6 shows a series of separations performed onchip in which increasing amounts of Th in a sample were added to a fixed amount of $\mathrm{Th}^{*}$ and $\mathrm{Ab}$. The separation occurs in less than 1 minute and the complex is well resolved from free $\mathrm{Th}^{*}$.

Data as in Figure 6 provides a calibration curve, such as is shown in Figure 7. Competitive assays of this type are well known to give non-linear calibration curves, but are extensively utilized in clinical diagnostics. To meet therapeutic criteria, serum samples should contain $\mathrm{Th}$ in the range of $10-20 \mu \mathrm{g} / \mathrm{ml}$. The lower axis shows the original sample concentrations before dilution, and it is clear that high sensitivity is attained in the therapeutic range. It is common to dilute the serum sample before analysis, and in this study we used a 200 -fold dilution factor. The upper axis shows the actual concentration in the measured samples, after dilution.

In a conventional assay one would follow the appearance of

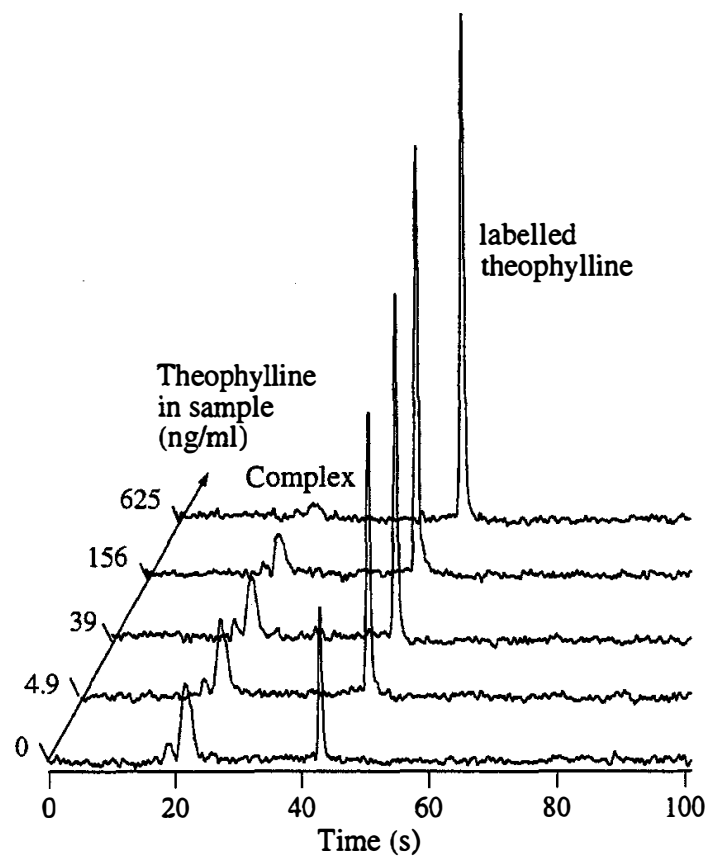

Figure 6 Competitive immunoassay of theophylline performed on-chip. Peaks for the complex and for free Th* are mixed. The ratio of peak areas changes as Th from a sample is added.

\section{Measurement concentration (ng/ml)}

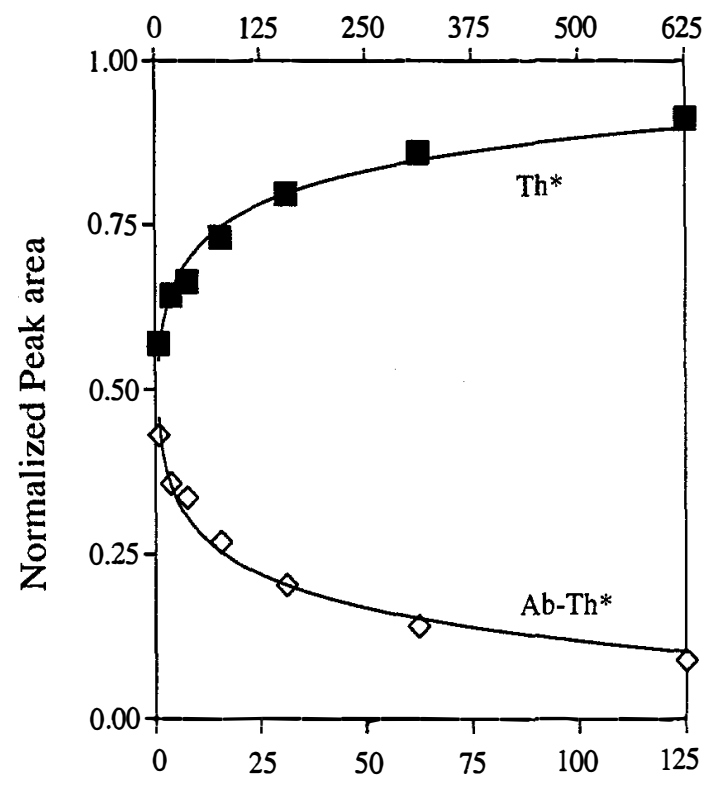

Theophylline in undiluted samples $(\mu \mathrm{g} / \mathrm{ml})$

Figure 7 Calibration curve for unlabelled Th in a sample. Lower axis shows concentrations before dilution, upper axis shows concentrations after dilution. 
free Th*. However, in this microchip format it is possible to observe both disappearance of the complex and appearance of free Th*. This process leads to greater precision of measurement. Both traces are shown in Figure 7. By measuring both components and recognizing that the total fluorescence signal should remain a constant, it is possible to normalize the signals. This compensates for small variations in the amount of material injected and gives better precision. The $y$-axis in Figure 7 is thus a plot of peak area for one of the two fluorescent species ratioed to the total fluorescent signal.

The calibration curve illustrates that we are readily able to measure theophylline in the therapeutic range, and that the absolute concentrations measured are in the $\mathrm{ng} / \mathrm{ml}$ range. The precision of the measurements is within $5 \%$, indicating good analytical performance.

\section{CONCLUSIONS}

Separation of proteins, in particular strongly adsorbent proteins such as immunoglobulins, offers significant difficulty for capillary electrophoresis methods $[2,3]$. Demonstration that it can be accomplished on-chip, for a variety of native-form IgG's from different sources is a significant step in developing major applications for CE-based microfluidic systems.

In this work we have shown that separations of a large variety of reactants and products created by immunological reactions can be performed on-chip. The antigens evaluated cover a range from very large proteins $(160,000$ Dalton $\mathrm{IgG})$ to very small molecules (180 Dalton theophylline). Also, several types of procedures were performed effectively: the direct assay of the antigen human IgG, where the antibody is labelled; the direct assay of an antibody where the antigen is labelled; and the competitive assay of a drug, where a certain amount of the drug is labelled. The assays are highly selective. In the case of the anti-BSA determination, this protein is easily determined in the presence of many others. For the theophylline assay, the drug is determined at low concentration in blood serum, which is a complex mixture of many components. Applications for the assays described range from the determination of antibody production in a fermentation process to analysis of drugs or infectious diseases, indicating the vast array of fields these devices could be applied to.

When combined with our previous studies of pre- and postseparation reactions on-chip $[1,4]$, it is clear that it will be possible to drive the complete process of antibody and sample mixing, incubation and separation within the confines of a chip. This offers the potential for a highly automated, rapid and easily programmed system. With a certain amount of engineering it is likely that portable systems incorporating these chips, using already existing light source, detection and power supply technologies, could be readily achieved.

\section{ACKNOWLEDGMENTS}

We thank DRES and NSERC of Canada, and Shimadzu for support. N.C. thanks the Alberta Microelectronic Centre for a graduate fellowship and use of their facilities.

\section{REFERENCES}

1. D.J. Harrison, K. Fluri, K. Seiler, Z. Fan, C.S. Effenhauser, A. Manz Science 261, 895 (1993).
2. N.M. Schultz, R.T. Kennedy Anal. Chem. 65, 3161 (1993).

3. K. Shimura, B. L. Karger Anal. Chem. 66, 9 (1994).

4. D.J. Harrison, K. Fluri, N. Chiem, T. Tang, Z. Fan Transducers 95 Technical Digest Vol 1 (1995) pp 752-755.

5. L.B. Koutny, D. Schmalzing, T.A. Taylot, M. Fuchs Anal. Chem. 68, 18 (1996).

6. Z. Fan, D. J. Harrison Anal. Chem. 66, 177 (1994).

7. M. Albin, R. Weinberger, E. Sapp, S. Moring, Anal. Chem. 63, 417 (1991). 